\title{
Statins-Treatment Option for Central Nervous System Autoimmune Disease?
}

\author{
Martin S. Weber,* Lawrence Steinman, ${ }^{\dagger}$ and Scott S. Zamvil* \\ *Department of Neurology and Program in Immunology, University of California, San Francisco, California 94143; \\ ${ }^{\dagger}$ Department of Neurology and Neurological Sciences and Interdepartmental Program in Immunology, Stanford University, \\ Stanford, California 94305
}

Summary: Statins, inhibitors of the enzyme 3-hydroxy-3methylglutaryl coenzyme A reductase, are well-established agents to lower cholesterol levels and prevent cardiovascular morbidity. Independent of their lipid-lowering properties, statins have been shown to exert pleiotropic immunomodulatory effects in various animal models of human autoimmune disease. In experimental autoimmune encephalomyelitis, a murine model for multiple sclerosis, statins prevented disease onset and even reversed paralysis when treatment was initiated after experimental autoimmune encephalomyelitis was fully estab- lished. Furthermore, well-tolerated oral statins were recently shown to exert synergistic benefit in experimental autoimmune encephalomyelitis in combination with existing agents for multiple sclerosis therapy. Based primarily on these encouraging results, statins are now being tested in clinical trials as a monotherapy for multiple sclerosis, as well as in combination with approved disease-modifying therapies. Key Words: Multiple sclerosis, immunomodulatory agents, statins, experimental autoimmune encephalomyelitis, novel therapy.

\section{INTRODUCTION}

Statins are oral inhibitors of the 3-hydroxy-3-methylglutaryl coenzyme A (HMG-CoA) reductase, which are widely prescribed for their cholesterol-lowering properties. HMG-CoA reductase is the rate-limiting enzyme that catalyzes the conversion of HMG-CoA to L-mevalonate, a key intermediate for cholesterol biosynthesis ${ }^{1}$ (FIG. 1). Because statins were first approved for treatment of hypercholesterolemia 20 years ago, these drugs have established themselves as very well-tolerated agents with rare side effects. More recent studies (primarily in animal models of autoimmune diseases) have revealed that besides their lipid-lowering properties, statins exert pleiotropic immunomodulatory effects that may benefit treatment of neuro-inflammatory disorders, such as multiple sclerosis (MS). Statins are particularly attractive agents for the treatment of MS, as all currently approved therapies of the Food and Drug Administration, such as interferon-beta, glatiramer acetate (GA), mitox-

Address correspondence and reprint requests to: Scott S. Zamvil, M.D., Ph.D., Associate Professor, Department of Neurology, Program in Immunology, University of California, San Francisco, 513 Parnassus Avenue, S-268, San Francisco, CA 94143. E-mail: zamvil@ucsf. neuroimmnunol.org. antrone, and natalizumab, which are only partially effective, administered parenterally, and have side effects.

\section{Immunomodulatory mechanism of action}

Besides cholesterol reduction, $\mathrm{HMG}-\mathrm{Co}$ A reductase regulates the production of isoprenoid intermediates in the branched mevalonate pathway. ${ }^{2}$ (FIG. 1). Numerous studies have investigated the role of these isoprenoids in inflammation associated with atherosclerosis and immune-mediated inflammatory conditions. The 15-carbon farnesyl pyrophosphate and the 20-carbon geranylgeranyl pyrophosphate participate in the post-translational modification of small regulatory proteins, a process known as isoprenylation. ${ }^{3}$ Isoprenylation of these proteins is necessary for their intracellular trafficking and anchoring at the cytoplasmic surface of the plasma membrane, where they function. Among proteins that undergo isoprenylation are members of the Ras family, Rho/Rac/ cell division cycle 42 (cdc42) and Rab families. These small proteins act as molecular switches, cycling between inactive GDP-bound and GTP-bound active states, where they control various signaling pathways. ${ }^{4}$ Ras proteins are farnesylated and primarily exert effects on cell differentiation and proliferation. Rho family proteins, which are geranylgeranylated, regulate cytoskeletal reor- 


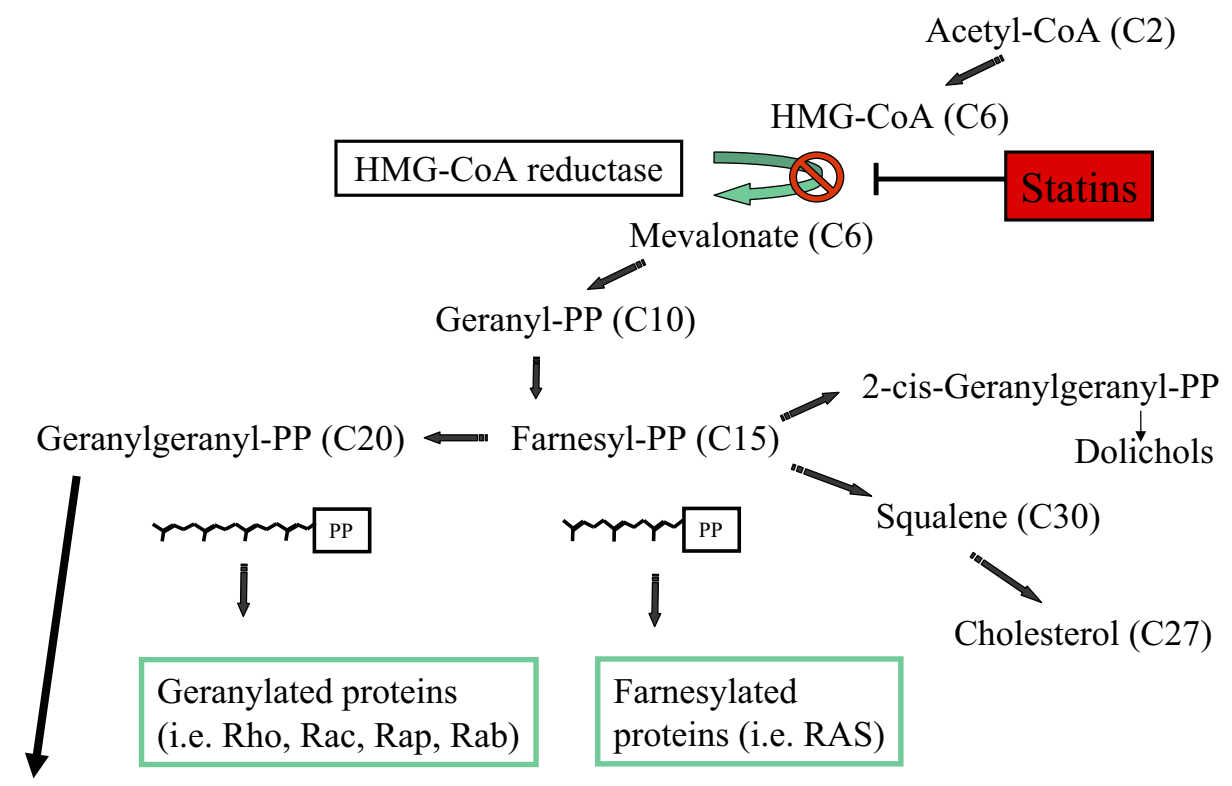

Ubiquinone

FIG. 1. Diagram of cholesterol biosynthesis and isoprenylation of regulatory proteins. Farnesylpyrophosphate (farnesyl-PP) and its derivative geranylgeranyl-PP are mediating posttranslational prenylation of regulatory proteins, such as Ras and Rho. Their prenylation permits membrane translocation and subsequent activation, which is crucial for various cellular functions, such as migration, differentiation, and proliferation. Statin-mediated inhibition of the 3-hydroxy-3-methylglutaryl coenzyme A (HMG-CoA) reductase not only decreases cholesterol biosynthesis, but also prevents isoprenylation and activation of regulatory proteins.

ganization. Furthermore, prenylation is required for members of the Rab family of GTP binding proteins, which are geranylgeranylated and involved in vesicular membrane trafficking. Thus, by inhibiting HMG-CoA reductase, statins not only inhibit the synthesis of cholesterol, but also limit availability of isoprenoid intermediates, which are required for activation of various cell types, including immune cells.

\section{Statins in treatment of experimental CNS autoimmunity}

Experimental autoimmune encephalomyelitis (EAE), a murine model for MS, is characterized by relapsing or chronic paralysis. In EAE, myelin-specific CD4+ T cells cause CNS inflammation ${ }^{5}$ which is associated with both demyelination and axonal damage. ${ }^{6}$ Daily statin treatment could not only prevent the onset of EAE, ${ }^{7-10}$ but also reverse clinical symptoms when treatment was initiated after paralysis was fully established. ${ }^{7,8}$ These studies demonstrated that statins interfere with the pathogenesis of EAE at various steps, including antigen presentation, activation and differentiation of myelinreactive $\mathrm{T}$ cells, and recruitment of leukocytes into the CNS. These pleiotropic statin effects on immune cell function and migration are discussed in the following sections.

\section{Modulation of antigen presentation}

Activation of $\mathrm{CD}^{+} \mathrm{T}$ cells requires recognition of linear peptide antigens presented that are in the context of the major histocompatibility complex (MHC) class II molecules expressed on the surface of antigen presenting cells (APC).

Kwak and colleagues ${ }^{11}$ demonstrated that atorvastatin inhibits upregulation of interferon (IFN)- $\gamma$-inducible MHC class II expression on endothelial cells as well as on monocytes. A later study reported an inhibitory effect of atorvastatin on IFN- $\gamma$-inducible MHC class II expression, including microglia, a residential APC population that may have a key role in antigen presentation within the CNS. ${ }^{7}$ Inhibition of MHC class II upregulation on microglia by atorvastatin appeared to be mediated through inhibition of the mevalonate pathway as it was reversed by addition of mevalonate. Similarly, inhibition of MHC class II expression in human endothelial cells by simvastatin was reversed by mevalonate and the 20 carbon geranylgeranyl pyrophosphate, but not by squalene, indicating an involvement of GTP-binding proteins in MHC class II inhibition. ${ }^{12}$

In addition to binding of the T-cell receptor to antigen presented in the context of the MHC class II molecule (signal 1), a second signal is required for efficient T-cell activation. ${ }^{13} \mathrm{CD} 40$ ligand, expressed on antigen-activated T cells express recognizes CD40 on the surface of APC. This interaction triggers expression of further co-stimulatory molecules on APC, such as B7-1 (CD80) and B7-2 (CD86), which subsequently interact with co-stimulatory molecules, such as CD28 on the surface of T cells (FIG. 2). In vitro, simvastatin and atorvastatin both prevented maturation of 


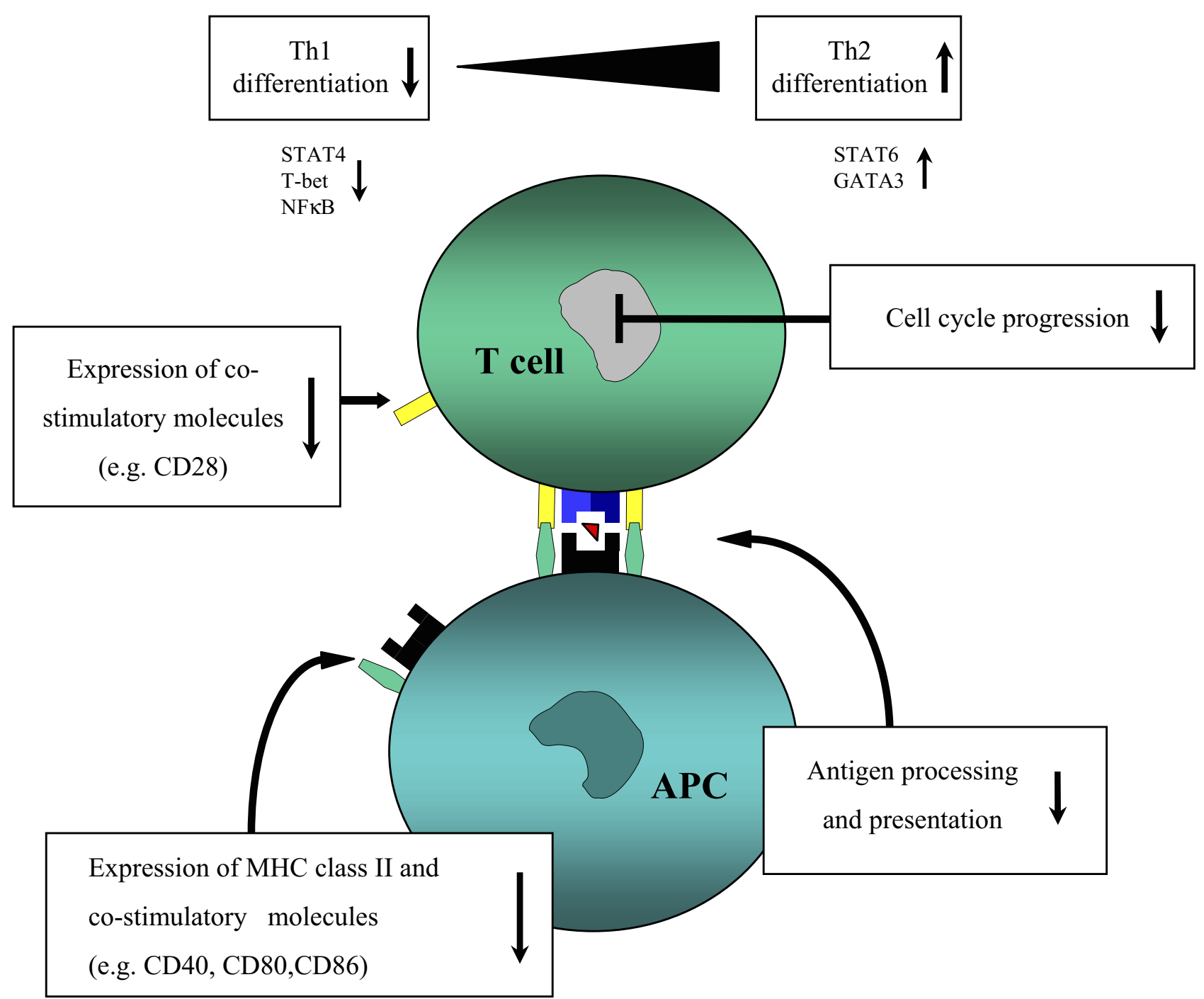

FIG. 2. Diagram of statin-mediated modulation of antigen presentation and activation of T cells. Statins inhibit expression of major histocompatibility complex (MHC) class II molecules and co-stimulatory molecules on antigen-presenting cells preventing antigen presentation to $\mathrm{CD} 4^{+} \mathrm{T}$ cells. They also exert an inhibitory effect on cell cycle progression, which directly inhibits T-cell proliferation. When activated, T-cell differentiation is deviated toward an anti-inflammatory Th2 phenotype. (APC = antigen presenting cells; GATA3

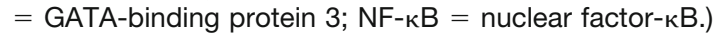

professional APC, which resulted in a strongly reduced ability to mediate T-cell proliferation. ${ }^{14}$ Activation of $\mathrm{T}$ cells could be restored by addition of either mevalonate or the 20-carbon geranylgeranyl pyrophosphate indicating that statins mediated inhibition of APC maturation through their effect on the mevalonate pathway. Similarly, atorvastatin inhibited IFN- $\gamma$-inducible expression of CD40, CD80, and CD86 molecules on microglial cells. ${ }^{7}$ In addition, atorvastatin treatment was found to reduce secretion of APCderived cytokines that are involved in the differentiation of $\mathrm{T}$ cells into pro-inflammatory Th 1 cells. ${ }^{15}$ Taken together, these data suggest that statins inhibit various aspects of APC-T cell interaction required for pro-inflammatory T-cell activation (FIG. 2).

\section{Modulation of T-lymphocyte proliferation and differentiation}

Independent of their effect on antigen presentation, statins may exert a direct inhibitory effect on T-cell proliferation as in vitro treatment of purified $\mathrm{T}$ cells could also inhibit proliferation when antigen was presented by untreated APC. ${ }^{7}$ Aktas and colleagues ${ }^{8}$ reported that this inhibitory effect on T-cell proliferation may be mediated through negative regulation of cellcycle progression consistent with inhibition of GTPasemediated cell proliferation. Subsequently, the same group demonstrated that atorvastatin-mediated phosphorylation of extracellular signal-regulated kinase 1 (ERK1) is responsible for T-cell anergy. ${ }^{16}$ 
$\mathrm{CD}^{+}{ }^{+} \mathrm{T}$ cells can be categorized based on the profile of cytokines that they secrete. ${ }^{17}$ Th1 cells have an important role in initiating EAE, providing pro-inflammatory cytokines such as IFN- $\gamma$, interleukin (IL)-2 and IL-12, and tumor necrosis factor. Another subset of proinflammatory $\mathrm{T}$ cells, IL-17-secreting Th17 cells was discovered and identified to have a key role in sustaining inflammation and tissue damage in CNS autoimmunity. ${ }^{18,19}$ In contrast, Th2 cells that secrete IL-4, IL-5, IL-10, and IL-13, have downregulatory properties on the inflammatory cascade in EAE, and are believed to have a beneficial role in regulation of MS. Similarly, $\mathrm{CD} 4+\mathrm{CD} 25+$ FoxP3 + regulatory $\mathrm{T}$ cells (Treg) may have a role in maintaining tolerance and preventing autoimmune disease and were recently described to be deficient in number and function in $\mathrm{MS}^{20}$

Several EAE studies indicate that statins suppress differentiation of myelin-specific $\mathrm{CD}^{+}{ }^{+} \mathrm{T}$ cells into proinflammatory Th1 cells and may promote Th2 differentiation. Statin treatment was associated with reduced phosphorylation (activation) of STAT4 (signal transducer and activator of transcription 4), which is required for IL-12-dependent Th1-differentiation and increased phosphorylation of STAT6, which is responsible for IL4-dependent Th2 differentiation. ${ }^{7}$ Nath and colleagues ${ }^{21}$ demonstrated that in vitro statins promoted T-cell expression of GATA-binding protein 3 (GATA3), a transcription factor associated with Th2 differentiation. In this report, statin treatment also exerted an inhibitory effect on the activation of nuclear factor- $\kappa \mathrm{B}(\mathrm{NF}-\kappa \mathrm{B})$ and T-bet, a T-box transcription factor associated with Th1 differentiation. ${ }^{22-24}$

Whereas suppression of pro-inflammatory Th1 responses is the most consistent finding throughout these animal studies, not all investigations have demonstrated a corresponding induction of Th2 cytokines. For example, lovastatin was found to ameliorate experimental autoimmune uveitis, another Th-1 mediated autoimmune disease model, which was associated with an inhibition of pro-inflammatory cytokines without a corresponding induction of a Th2 cytokine profile. ${ }^{25}$ Similarly, in collagen-induced arthritis, high-dose simvastatin suppressed collagen-specific Th1 humoral and cellular immune responses without an upregulation of Th2 cytokines. $^{26}$ These varying findings on the induction of Th2 cytokines under statin treatment may relate to differences in the model system, the statin used, and the dose. Alternatively, statin-mediated Th2 deviation might not be an active induction of anti-inflammatory Th2 cells, but rather an occurrence as a consequence of Th1 suppression. Whether statin treatment inhibits differentiation of pro-inflammatory Th17 cells and/or promotes development of Treg has not yet been reported.

\section{Modulation of adhesion molecule expression on leukocytes and endothelial}

Recruitment of leukocytes into the CNS occurs in a cascade of events, including chemoattraction, cell adhesion, extravasation, and proteolytic degradation of the extracellular matrix. The expression of adhesion molecules on both leukocytes and the endothelium plays an important role in the initiation of transmigration. The integrin leukocyte function antigen 1 is an adhesion molecule on lymphocytes that binds to intercellular adhesion molecule 1 (ICAM-1) expressed on the endothelium. Interestingly, both molecules have been identified to be expressed on infiltrating lymphocytes and endothelial cells in perivascular MS lesions. ${ }^{27}$ Some data suggest that lovastatin can bind directly to the extracellular domain of leukocyte function antigen 1 independent of inhibition of HMG-CoA reductase, which results in a reduced adhesion capacity of lymphocytes to ICAM-1. ${ }^{28}$ Correspondingly, macrophage adhesion molecule-1 (Mac-1) (CD11b/CD18) is the integrin expressed on monocytes binding to ICAM-1 on endothelial cells. In vitro, lovastatin was also shown to inhibit monocyte expression of Mac-1 and subsequent binding of these cells to the endothelium. ${ }^{29}$

On endothelial cells, statins were shown to decrease both constitutive and induced expression of ICAM-1, the key molecule for leukocyte adhesion. ${ }^{30,31}$ Furthermore, lovastatin was recently reported to inhibit expression of vascular cell adhesion molecule-1, another adhesion molecule expressed on endothelial cells, resulting in a significantly reduced transendothelial cell migration. ${ }^{32}$ Thus, statins inhibit recruitment of leukocytes into the CNS by suppressing expression of adhesion molecules on both activated leukocytes and brain endothelial cells. $^{33}$

\section{Inhibition of leukocyte migration and motility}

Independent of its role as an adhesion molecule, ICAM-1 engagement results in intracellular signaling responses that facilitate lymphocyte transendothelial migration. ${ }^{34-37}$ In this regard, it was shown that in vitro treatment of brain endothelial cells with lovastatin inhibited transendothelial T-cell migration ${ }^{10}$ consistent with the finding that Rho-GTPase is critically involved in transduction of ICAM-1-mediated signaling responses in brain endothelial cells, and consequently transendothelial migration of T lymphocytes. ${ }^{34,38}$

Once leukocytes crossed the endothelial barriers, they still have to traverse the basement membrane (basal lamina) of brain venuoles to access the CNS parenchyma. Due to their proteolytic properties, matrix metalloproteinases (MMPs) are physiologic mediators of cell migration through basement membranes and extracellular matrix. ${ }^{39}$ Two reports demonstrated that statins reduced secretion of MMP-9 by monocytes in a dose-dependent 
manner, which was reversed by the simultaneous addition of exogenous mevalonate. ${ }^{40,41}$ Specifically, secretion of MMP-9 from the monocytic cell line THP-1 could be inhibited by an inhibitor selective for geranylgeranyl transferase pointing to Rho being involved in the inhibitory process. ${ }^{42}$ Inhibition of monocyte MMP-9 secretion by cerivastatin in contrary was reversible by farnesyl pyrophosphate (FPP), thus implicating a role for Ras. ${ }^{40}$ These data show that multiple small GTPase signaling pathways may be involved in MMP-9 production and as such may represent the mechanism by which statins inhibit their secretion.

\section{Statins may be a treatment option for multiple sclerosis}

The immunomodulatory effects of statins observed in animal models of inflammatory diseases provided the impetus for several clinical trials. In a first small openlabel study, seven relapsing-remitting MS patients with an active disease course received $20 \mathrm{mg}$ lovastatin per day. ${ }^{43} \mathrm{~A}$ brain MRI revealed decreased inflammation under lovastatin treatment, although no significant clinical changes were observed in this small cohort. In another small open-label trial, simvastatin was tested in patients with clinically definite relapsing-remitting MS. ${ }^{44}$ Patients with at least one gadolinium-enhancing CNS lesion in the 3-month pre-treatment period were treated with $80 \mathrm{mg}$ simvastatin daily for 6 months. Posttreatment MRI indicated a decrease of approximately $45 \%$ in the mean number and volume of gadoliniumenhancing lesions in treated subjects. Although these preliminary data are encouraging, these reports need to be interpreted cautiously. The small number of patients treated in this trial was selected on the basis of having comparably active brain MRIs at the time of enrollment, so that the observed decreased inflammatory activity under statin treatment may reflect the regression to the mean. To address this issue, a larger, 152-patient, placebo-controlled trial (see http://immunetolerance.org/staycis/) is currently being conducted, and the results are eagerly being awaited. In this trial, patients who have experienced a first CNS demyelinating attack (i.e., a "clinically isolated syndrome") are treated for 12 months with $80 \mathrm{mg}$ atorvastatin in 14 centers in North America. The primary endpoint of this study is met either by a further clinical exacerbation resulting in clinically definite relapsing-remitting MS, or more than three new T2 or gadolinium-enhancing MRI brain lesions. A clinical trial testing atorvastatin for the treatment of rheumatoid arthritis obtained encouraging data for this Th1-mediated organ-specific autoimmune disease. ${ }^{45}$ In addition to their ongoing disease-modifying anti-rheumatic drug therapy, rheumatoid arthritis patients were treated with $40 \mathrm{mg}$ atorvastatin or placebo daily. Compared with the active placebo arm, atorvastatin treatment mediated clinical im- provement that was associated with a decreased level of inflammation markers.

Current trials may determine that oral statins are only partially effective as monotherapy in the treatment of MS. If so, they might prove to be useful in combination with existing parenteral disease-modifying medications. For instance, GA is a well-tolerated polypeptide-based therapy approved for relapsing-remitting MS that appears to preferentially cause a Th2 deviation of myelinspecific T cells. ${ }^{46,47}$ Furthermore, more recent data indicate that GA treatment mediates immunomodulatory activity on APC, promoting the secretion of anti-inflammatory cytokines and inhibiting the secretion of proinflammatory cytokines. ${ }^{48,49}$ In EAE, the combination of GA and atorvastatin synergistically ameliorated established paralysis, ${ }^{15}$ which was associated with an enhanced differentiation of $\mathrm{T}$ cells into anti-inflammatory Th2 cells. In vitro experiments revealed that this combination also altered the cytokine profile of activated monocytes in an additive manner. Based primarily on these findings, clinical trials testing atorvastatin in combination with GA are being anticipated. Encouraging results were also obtained from testing the combination of atorvastatin and 5-aminoimidazole-4-carboxamide-1beta-D-ribofuranoside, an immunomodulating agent that activates AMP-activated protein kinase. ${ }^{50}$ This combination was an additive for EAE prevention and reduced cellular infiltration of the CNS more than either drug alone. In addition, this combination lessened axonal loss, which might be of particular interest for treatment of MS at the later neurodegenerative stage. A recent study suggests that the combination of atorvastatin and high-dose interferon beta 1a (Rebif Serono, Geneva, Switzerland), another approved drug for MS treatment, may however not be of clinical benefit. Patients administering interferon beta 1a for at least 6 months were randomized in a double-blind fashion to one of three groups: 1) daily placebo, 2) daily medium dose atorvastatin (40 mg per day), or 3) daily high-dose atorvastatin (80 $\mathrm{mg}$ per day) as add on therapy. When the clinical and imaging data for this trial were combined, the analysis indicated that this combination may be antagonistic and lead to paradoxically increased MS activity. ${ }^{51}$ However, these data must be viewed cautiously as they were obtained from a small single-center study. A multicenter, rater-blinded, parallel group study is currently testing the combination of $40 \mathrm{mg}$ of atorvastatin and interferon beta $1 \mathrm{~b}$ in 80 relapsing-remitting MS patients. Results from this study and further studies that are continuing to treat MS patients with the combination of statins and interferon-beta are anticipated.

\section{Statins in treatment of other neurological disorders}

Independent of their effect on immune cells, statins may directly have neuroprotective effects on neuronal 
and glial cells that may extend their applicability to treatment of noninflammatory neurological conditions. In vitro, statins protect neurons from excitotoxic death caused by brain ischemia. ${ }^{52}$ As this neuroprotective effect was substantially attenuated by co-treatment with either mevalonate or cholesterol, it may be directly related to statin-mediated inhibition of neuronal cholesterol biosynthesis. Furthermore, lovastatin inhibited degeneration of oligodendrocyte progenitors, ${ }^{53}$ a process that is believed to contribute to impaired remyelination after myelin sheath damage. Indeed, lovastatin treatment restored remyelination in the spinal cord of mice with EAE, which was associated with increased expression of myelin proteins and transcription factors involved in differentiating oligodendrocytes. ${ }^{53}$

Furthermore, statin use might reduce prevalence or severity of Alzheimer's disease (AD).$^{54,55}$ In a small proof-of-concept study, patients with mild to moderate $\mathrm{AD}$ received $80 \mathrm{mg}$ atorvastatin daily for 1 year and improved significantly on the AD Assessment Scale. ${ }^{56}$ Various statin-mediated effects might account for this clinical benefit. In an animal model of $\mathrm{AD}$, cholesterol was found to facilitate the production of amyloid $\beta$, the putative neurotoxic precipitate in $\mathrm{AD} .{ }^{57,58} \mathrm{In}$ another model, in vivo treatment with simvastatin reduced cholesterol and the production of amyloid $\beta$. Taken together, these data indicate that statins might inhibit accumulation of amyloid $\beta$ in CNS plaques by inhibition of cholesterol biosynthesis. Although AD is a neurodegenerative disease, there is evidence that inflammation might contribute to its pathogenesis. ${ }^{59}$ In addition, statins might prove to be beneficial in the treatment of $\mathrm{AD}$, owing to their newly discovered anti-inflammatory properties.

There is also strong evidence that several properties of statins may contribute to preventing an ischemic stroke. As previously described, statins reduce the secretion of proteolytic MMPs, lower expression of endothelial adhesion molecules, and inhibit activation of immune cells, factors which may facilitate the rupture of an existing atherosclerotic plaque, ${ }^{60}$ resulting in an atherogenic thrombus. Statins enhance endothelial NOS, leading to relaxation of the vascular bed, reduced proliferation of vascular smooth muscle, and inhibition of thrombocyte aggregation. ${ }^{61}$ Statins appear to directly deactivate platelets, which may further reduce the risk for a thrombus. ${ }^{62}$ In experimental CNS ischemia, immediately initiated statin treatment resulted in an improved clinical outcome. ${ }^{63}$ In patients with known ischemic heart disease, statins prevented the occurrence of a secondary ischemic stroke by $21 \% .{ }^{64}$ In individuals with high-risk factors to developing a stroke, such as hypertension or diabetes, statin treatment also reduced the incidence of a first stroke. ${ }^{65}$ To determine whether statins can prevent a stroke in patients without any of these predispositions, a large clinical trial with 4700 patients randomized to ei- ther $80 \mathrm{mg}$ atorvastatin or a placebo is currently underway. ${ }^{66}$

\section{Adverse effects of statins}

Although generally considered safe and well-tolerated drugs, statins do have side effects. Statins are metabolized by the cytochrome P450 pathway, thereby occasionally causing hepatotoxicity $(<3 \%$ of patients), predominantly with a reversible elevation of transaminases. This side effect should be particularly considered when statins are administered in combination with other agents metabolized through the cytochrome $\mathrm{P} 450$ pathway. Myopathy is a rare (less than $0.2 \%),{ }^{67-69}$ but potentially severe adverse event, as it can lead to rhabdomyolysis (less than $0.05 \%$ ) and even kidney failure. ${ }^{70}$ Again, rhabdomyolysis is more likely to occur when statins are used with other drugs, especially lipid-lowering fibrates, and with compounds that are similarly metabolized by the cytochrome P450 pathway. Another side effect occasionally reported in association with statin treatment is idiopathic polyneuropathy. However, a comparative study revealed that the relative risk for a polyneuropathy to occur under statin treatment was only slightly higher than in the hyperlipidemia nontreated cohort or the general population. ${ }^{71,72}$

\section{CONCLUSIONS}

Besides their lipid-lowering properties, statins exert pleiotropic immunomodulatory effects that may be of therapeutic benefit in CNS autoimmune disease. In murine EAE, statins have shown to target multiple key elements of the immunologic cascade that is believed to lead to inflammatory infiltration and tissue damage in MS. Recent data indicate that statins mediate the majority of their anti-inflammatory properties through inhibition of an alternative branch of the mevalonate pathway, which is primarily recognized for its product, cholesterol. To date, two small open-label trials have tested oral statin treatment in relapsing-remitting MS and reported encouraging preliminary results. Based on these findings, the outcome of a larger placebo-controlled trial testing atorvastatin in early MS is being awaited with high expectations.

\section{REFERENCES}

1. Ginsberg HN. Effects of statins on triglyceride metabolism. Am J Cardiol 1998;81:32B-35B.

2. Goldstein JL, Brown MS. Regulation of the mevalonate pathway. Nature 1990;343:425-430.

3. Zhang FL, Casey PJ. Protein prenylation: molecular mechanisms and functional consequences. Annu Rev Biochem 1996;65:241269.

4. Takai Y, Sasaki T, Matozaki T. Small GTP-binding proteins. Physiol Rev 2001;81:153-208.

5. Zamvil SS, Steinman L. The T lymphocyte in experimental allergic encephalomyelitis. Ann. Rev. Immunol 1990;8:579-621. 
6. Kornek B, Storch MK, Weissert R, et al. Multiple sclerosis and chronic autoimmune encephalomyelitis: a comparative quantitative study of axonal injury in active, inactive, and remyelinated lesions. Am J Pathol 2000;157:267-276.

7. Youssef S, Stuve O, Patarroyo JC, et al. The HMG-CoA reductase inhibitor, atorvastatin, promotes a Th2 bias and reverses paralysis in central nervous system autoimmune disease. Nature 2002;420: $78-84$.

8. Aktas O, Waiczies S, Smorodchenko A, et al. Treatment of relapsing paralysis in experimental encephalomyelitis by targeting Th1 cells through atorvastatin. J Exp Med 2003;197:725-733.

9. Stanislaus R, Gilg AG, Singh AK, Singh I. Immunomodulation of experimental autoimmune encephalomyelitis in the Lewis rats by Lovastatin. Neurosci Lett 2002;333:167-170.

10. Greenwood J, Walters CE, Pryce G, et al. Lovastatin inhibits brain endothelial cell Rho-mediated lymphocyte migration and attenuates experimental autoimmune encephalomyelitis. Faseb J 2003; 17:905-907.

11. Kwak B, Mulhaupt F, Myit S, Mach F. Statins as a newly recognized type of immunomodulator. Nat Med 2000;6:1399-1402.

12. Sadeghi MM, Tiglio A, Sadigh K, et al. Inhibition of interferongamma-mediated microvascular endothelial cell major histocompatibility complex class II gene activation by HMG-CoA reductase inhibitors. Transplantation 2001;71:1262-1268.

13. Dustin ML, Shaw AS. Costimulation: building an immunological synapse. Science 1999;283:649-650.

14. Yilmaz A, Reiss C, Tantawi O, Weng A, Stumpf C, Raaz D, et al. HMG-CoA reductase inhibitors suppress maturation of human dendritic cells: new implications for atherosclerosis. Atherosclerosis 2004;172:85-93.

15. Stuve O, Youssef S, Weber MS, et al. Immunomodulatory synergy by combination of atorvastatin and glatiramer acetate in treatment of CNS autoimmunity. J Clin Invest 2006;116:1037-1044.

16. Waiczies S, Prozorovski T, Infante-Duarte C, et al. Atorvastatin induces $\mathrm{T}$ cell anergy via phosphorylation of ERK1. J Immunol 2005;174:5630-5635.

17. Abbas AK, Murphy KM, Sher A. Functional diversity of helper T lymphocytes. Nature 1996;383:787-793.

18. Bettelli E, Carrier Y, Gao W, et al. Reciprocal developmental pathways for the generation of pathogenic effector TH17 and regulatory T cells. Nature 2006;441:235-238.

19. Steinman L. A brief history of $\mathrm{T}(\mathrm{H}) 17$, the first major revision in the $\mathrm{T}(\mathrm{H}) 1 / \mathrm{T}(\mathrm{H}) 2$ hypothesis of $\mathrm{T}$ cell-mediated tissue damage. Nat Med 2007;13:139-145.

20. Viglietta V, Baecher-Allan C, Weiner HL, Hafler DA. Loss of functional suppression by $\mathrm{CD} 4+\mathrm{CD} 25+$ regulatory $\mathrm{T}$ cells in patients with multiple sclerosis. J Exp Med 2004;199:971-979.

21. Nath N, Giri S, Prasad R, Singh AK, Singh I. Potential targets of 3-hydroxy-3-methylglutaryl coenzyme a reductase inhibitor for multiple sclerosis therapy. J Immunol 2004;172:1273-1286.

22. Ho IC, Glimcher LH. Transcription: tantalizing times for T cells. Cell 2002;109(Suppl):S109-120.

23. Murphy KM, Reiner SL. The lineage decisions of helper T cells. Nat Rev Immunol 2002;2:933-944.

24. Robinson DS, O'Garra A. Further checkpoints in Th1 development. Immunity 2002;16:755-758.

25. Gegg ME, Harry R, Hankey D, et al. Suppression of autoimmune retinal disease by lovastatin does not require Th2 cytokine induction. J Immunol 2005;174:2327-2335.

26. Leung BP, Sattar N, Crilly A, et al. A novel anti-inflammatory role for simvastatin in inflammatory arthritis. J Immunol 2003;170: $1524-1530$.

27. Cannella B, Raine CS. The adhesion molecule and cytokine profile of multiple sclerosis lesions. Ann Neurol 1995;37:424-435.

28. Weitz-Schmidt G, Welzenbach K, Brinkmann V, et al. Statins selectively inhibit leukocyte function antigen-1 by binding to a novel regulatory integrin site. Nat Med 2001;7:687-692.

29. Weber C, Erl W, Weber KS, Weber PC. HMG-CoA reductase inhibitors decrease $\mathrm{CD} 11 \mathrm{~b}$ expression and $\mathrm{CD} 11 \mathrm{~b}$-dependent adhesion of monocytes to endothelium and reduce increased adhesiveness of monocytes isolated from patients with hypercholesterolemia. J Am Coll Cardiol 1997;30:1212-1217.
30. Chung HK, Lee IK, Kang H, et al. Statin inhibits interferongamma-induced expression of intercellular adhesion molecule-1 (ICAM-1) in vascular endothelial and smooth muscle cells. Exp Mol Med 2002;34:451-461.

31. Doo YC, Han SJ, Han SW, et al. Effect of preexisting statin use on expression of C-reactive protein, adhesion molecules, interleukin-6, and antioxidized low-density lipoprotein antibody in patients with unstable angina undergoing coronary stenting. Clin Cardiol 2005;28:72-76.

32. Prasad R, Giri S, Nath N, Singh I, Singh AK. Inhibition of phosphoinositide 3 kinase-Akt (protein kinase B)-nuclear factor-kappa B pathway by lovastatin limits endothelial-monocyte cell interaction. J Neurochem 2005;94:204-214.

33. Greenwood J, Steinman L, Zamvil S. Statin therapy in autoimmunity: From protein prenylation to immunomodulation. Nat Rev Immunol 2006;6:358.

34. Etienne S, Adamson P, Greenwood J, Strosberg AD, Cazaubon S, Couraud PO. ICAM-1 signaling pathways associated with Rho activation in microvascular brain endothelial cells. J Immunol 1998;161:5755-5761.

35. Adamson P, Etienne S, Couraud PO, Calder V, Greenwood J. Lymphocyte migration through brain endothelial cell monolayers involves signaling through endothelial ICAM-1 via a rho-dependent pathway. J Immunol 1999;162:2964-2973.

36. Etienne-Manneville S, Manneville JB, Adamson P, Wilbourn B, Greenwood J, Couraud PO. ICAM-1-coupled cytoskeletal rearrangements and transendothelial lymphocyte migration involve intracellular calcium signaling in brain endothelial cell lines. J Immunol 2000;165:3375-3383.

37. Adamson P, Wilbourn B, Etienne-Manneville S, et al. Lymphocyte trafficking through the blood-brain barrier is dependent on endothelial cell heterotrimeric G-protein signaling. Faseb J 2002;16: 1185-1194.

38. Walters CE, Pryce G, Hankey DJ, et al. Inhibition of Rho GTPases with protein prenyltransferase inhibitors prevents leukocyte recruitment to the central nervous system and attenuates clinical signs of disease in an animal model of multiple sclerosis. J Immunol 2002;168:4087-4094.

39. Yong VW, Krekoski CA, Forsyth PA, Bell R, Edwards DR. Matrix metalloproteinases and diseases of the CNS. Trends Neurosci 1998;21:75-80.

40. Ganne F, Vasse M, Beaudeux JL, et al. Cerivastatin, an inhibitor of HMG-CoA reductase, inhibits urokinase/urokinase-receptor expression and MMP-9 secretion by peripheral blood monocytes-a possible protective mechanism against atherothrombosis. Thromb Haemost 2000;84:680-688.

41. Bellosta S, Via D, Canavesi M, et al. HMG-CoA reductase inhibitors reduce MMP-9 secretion by macrophages. Arterioscler Thromb Vasc Biol 1998;18:1671-1678.

42. Wong B, Lumma WC, Smith AM, Sisko JT, Wright SD, Cai TQ. Statins suppress THP-1 cell migration and secretion of matrix metalloproteinase 9 by inhibiting geranylgeranylation. J Leukoc Biol 2001;69:959-962.

43. Sena A, Pedrosa R, Graca Morais M. Therapeutic potential of lovastatin in multiple sclerosis. J Neurol 2003;250:754-755.

44. Vollmer T, Key L, Durkalski V, et al. Oral simvastatin treatment in relapsing-remitting multiple sclerosis. Lancet 2004;363:16071608.

45. McCarey DW, McInnes IB, Madhok R, et al. Trial of Atorvastatin in Rheumatoid Arthritis (TARA): double-blind, randomised placebo-controlled trial. Lancet 2004;363:2015-2021.

46. Neuhaus O, Farina C, Wekerle H, Hohlfeld R. Mechanisms of action of glatiramer acetate in multiple sclerosis. Neurology 2001; 56:702-708.

47. Duda PW, Schmied MC, Cook SL, Krieger JI, Hafler DA. Glatiramer acetate (Copaxone) induces degenerate, Th2-polarized immune responses in patients with multiple sclerosis. J Clin Invest 2000;105:967-976.

48. Kim HJ, Ifergan I, Antel JP, et al. Type 2 monocyte and microglia differentiation mediated by glatiramer acetate therapy in patients with multiple sclerosis. J Immunol 2004;172:7144-7153. 
49. Weber MS, Starck M, Wagenpfeil S, Meinl E, Hohlfeld R, Farina C. Multiple sclerosis: glatiramer acetate inhibits monocyte reactivity in vitro and in vivo. Brain 2004;127(Pt 6):1370-1378.

50. Paintlia AS, Paintlia MK, Singh I, Singh AK. Immunomodulatory effect of combination therapy with lovastatin and 5-aminoimidazole-4-carboxamide-1-beta-D-ribofuranoside alleviates neurodegeneration in experimental autoimmune encephalomyelitis. Am J Pathol 2006;169:1012-1025.

51. Birnbaum GA, I. A double-blind, placebo controlled trial of atorvastatin in combination with subcutaneous interferon-beat $1 \mathrm{a}$ in persons with multiple sclerosis. Neurology 2007;68:A206.

52. Zacco A, Togo J, Spence K, et al. 3-hydroxy-3-methylglutaryl coenzyme A reductase inhibitors protect cortical neurons from excitotoxicity. J Neurosci 2003;23:11104-11111.

53. Paintlia AS, Paintlia MK, Khan M, Vollmer T, Singh AK, Singh I. HMG-CoA reductase inhibitor augments survival and differentiation of oligodendrocyte progenitors in animal model of multiple sclerosis. Faseb J 2005;19:1407-1421.

54. Wolozin B, Kellman W, Ruosseau P, Celesia GG, Siegel G. Decreased prevalence of Alzheimer disease associated with 3-hydroxy-3-methyglutaryl coenzyme A reductase inhibitors. Arch Neurol 2000;57:1439-1443.

55. Jick H, Zornberg GL, Jick SS, Seshadri S, Drachman DA. Statins and the risk of dementia. Lancet 2000;356:1627-1631.

56. Sparks DL, Sabbagh MN, Connor DJ, et al. Atorvastatin for the treatment of mild to moderate Alzheimer disease: preliminary results. Arch Neurol 2005;62:753-757.

57. Sparks DL, Scheff SW, Hunsaker JC, 3rd, Liu H, Landers T, Gross DR. Induction of Alzheimer-like beta-amyloid immunoreactivity in the brains of rabbits with dietary cholesterol. Exp Neurol 1994; 126:88-94.

58. Refolo LM, Malester B, LaFrancois J, et al. Hypercholesterolemia accelerates the Alzheimer's amyloid pathology in a transgenic mouse model. Neurobiol Dis 2000;7:321-331.

59. Akiyama H, Barger S, Barnum S, et al. Inflammation and Alzheimer's disease. Neurobiol Aging 2000;21:383-421.

60. Crisby M, Nordin-Fredriksson G, Shah PK, Yano J, Zhu J, Nilsson J. Pravastatin treatment increases collagen content and decreases lipid content, inflammation, metalloproteinases, and cell death in human carotid plaques: implications for plaque stabilization. Circulation 2001;103:926-933.

61. Endres M, Laufs U, Huang Z, et al. Stroke protection by 3-hy- droxy-3-methylglutaryl (HMG)-CoA reductase inhibitors mediated by endothelial nitric oxide synthase. Proc Natl Acad Sci U S A 1998;95:8880-8885.

62. Notarbartolo A, Davi G, Averna M, et al. Inhibition of thromboxane biosynthesis and platelet function by simvastatin in type IIa hypercholesterolemia. Arterioscler Thromb Vasc Biol 1995;15: 247-251.

63. Chen J, Zhang ZG, Li Y, et al. Statins induce angiogenesis, neurogenesis, and synaptogenesis after stroke. Ann Neurol 2003;53: $743-751$.

64. Amarenco P, Tonkin AM. Statins for stroke prevention: disappointment and hope. Circulation 2004;109(23 Suppl 1):III44-49.

65. Sever PS, Dahlof B, Poulter NR, et al. Prevention of coronary and stroke events with atorvastatin in hypertensive patients who have average or lower-than-average cholesterol concentrations, in the Anglo-Scandinavian Cardiac Outcomes Trial--Lipid Lowering Arm (ASCOT-LLA): a multicentre randomised controlled trial. Lancet 2003;361:1149-1158.

66. Amarenco P. Effect of statins in stroke prevention. Curr Opin Lipidol 2005;16:614-618.

67. Randomised trial of cholesterol lowering in 4444 patients with coronary heart disease: the Scandinavian Simvastatin Survival Study (4S). Lancet 1994;344:1383-1389.

68. Jukema JW, Bruschke AV, van Boven AJ, et al. Effects of lipid lowering by pravastatin on progression and regression of coronary artery disease in symptomatic men with normal to moderately elevated serum cholesterol levels. The Regression Growth Evaluation Statin Study (REGRESS). Circulation 1995;91:2528-2540.

69. Begolka WS, Vanderlugt CL, Rahbe SM, Miller SD. Differential expression of inflammatory cytokines parallels progression of central nervous system pathology in two clinically distinct models of multiple sclerosis. J Immunol 1998;161:4437-4446.

70. Graham DJ, Staffa JA, Shatin D, et al. Incidence of hospitalized rhabdomyolysis in patients treated with lipid-lowering drugs. JAMA 2004;292:2585-2590.

71. Gaist D, Jeppesen U, Andersen M, Garcia Rodriguez LA, Hallas J, Sindrup SH. Statins and risk of polyneuropathy: a case-control study. Neurology 2002;58:1333-1337.

72. Gaist D, Garcia Rodriguez LA, Huerta C, Hallas J, Sindrup SH. Are users of lipid-lowering drugs at increased risk of peripheral neuropathy? Eur J Clin Pharmacol 2001;56:931-933. 